\title{
BMJ Open How different are baby-led weaning and conventional complementary feeding? A cross-sectional study of infants aged 6-8 months
}

\author{
Brittany J Morison, ${ }^{1}$ Rachael W Taylor, ${ }^{2}$ Jillian J Haszard, ${ }^{1}$ Claire J Schramm, ${ }^{1}$ \\ Liz Williams Erickson, ${ }^{1}$ Louise J Fangupo, ${ }^{1}$ Elizabeth A Fleming, ${ }^{1}$ Ashley Luciano, ${ }^{1}$ \\ Anne-Louise $\mathrm{M}$ Heath ${ }^{1}$
}

To cite: Morison BJ, Taylor RW, Haszard JJ, et al. How different are baby-led weaning and conventional complementary feeding? A cross-sectional study of infants aged 6-8 months. BMJ Open 2016;6:e010665. doi:10.1136/bmjopen-2015010665

- Prepublication history for this paper is available online To view these files please visit the journal online (http://dx.doi.org/10.1136/ bmjopen-2015-010665)

Received 25 November 2015 Revised 26 March 2016 Accepted 19 April 2016

CrossMark

${ }^{1}$ Departments of Human Nutrition, University of Otago, Dunedin, New Zealand ${ }^{2}$ Department of Medicine, University of Otago, Dunedin, New Zealand

Correspondence to Dr Anne-Louise M Heath; anne-louise.heath@otago.ac. $\mathrm{nz}$

\section{ABSTRACT}

Objectives: To compare the food, nutrient and 'family meal' intakes of infants following baby-led weaning (BLW) with those of infants following a more traditional spoon-feeding (TSF) approach to complementary feeding.

Study design and participants: Cross-sectional study of dietary intake and feeding behaviours in 51 age-matched and sex-matched infants ( $\mathrm{n}=25 \mathrm{BLW}$, 26 TSF) 6-8 months of age.

Methods: Parents completed a questionnaire, and weighed diet records (WDRs) on 1-3 non-consecutive days, to investigate food and nutrient intakes, the extent to which infants were self-fed or parent-fed, and infant involvement in 'family meals'.

Results: BLW infants were more likely than TSF infants to have fed themselves all or most of their food when starting complementary feeding ( $67 \%$ vs $8 \%, p<0.001)$. Although there was no statistically significant difference in the large number of infants consuming foods thought to pose a choking risk during the WDR $(78 \%$ vs $58 \%$, $\mathrm{p}=0.172$ ), the $\mathrm{Cl}$ was wide, so we cannot rule out increased odds with BLW (OR, 95\% Cl: 2.57, 0.63 to 10.44). No difference was observed in energy intake, but BLW infants appeared to consume more total (48\% vs $42 \%$ energy, $p<0.001$ ) and saturated $(22 \%$ vs $18 \%$ energy, $p<0.001$ ) fat, and less iron ( $1.6 \mathrm{vs} 3.6 \mathrm{mg}$, $p<0.001$ ), zinc ( 3.0 vs $3.7 \mathrm{mg}, \mathrm{p}=0.001$ ) and vitamin $B_{12}(0.2$ vs $0.5 \mu \mathrm{g}, \mathrm{p}<0.001)$ than TSF infants. BLW infants were more likely to eat with their family at lunch and at the evening meal (both $p \leq 0.020$ ).

Conclusions: Infants following BLW had similar energy intakes to those following TSF and were eating family meals more regularly, but appeared to have higher intakes of fat and saturated fat, and lower intakes of iron, zinc and vitamin $B_{12}$. A high proportion of both groups were offered foods thought to pose a choking risk.

\section{INTRODUCTION}

Traditionally, parents have been advised to spoon feed their infant puréed foods from 'around' 6 months of age, progressing to

\section{Strengths and limitations of this study}

- First study to compare the dietary intake of children following baby-led weaning (BLW) with that of infants following a more traditional spoon-feeding (TSF) approach to complementary feeding.

- Weighed diet records with careful recording of foods offered and foods eaten.

- Age and sex matching of children following BLW and those following TSF.

- Small sample size.

- Participants defined themselves as following BLW or TSF.

mashed, then chopped foods, so that they are eating family foods by 12 months of age. ${ }^{1}{ }^{2}$ However, anecdotal reports suggest that an alternative method of complementary feeding, known as baby-led weaning (BLW), is becoming popular in New Zealand, the UK and Canada. In BLW, infants are not spoon fed at all, but instead feed themselves whole pieces of food, preferably from the family meal, from the onset of complementary feeding. ${ }^{34}$

Proponents of this baby-led approach suggest that it allows the infant to be in control of how much food they eat, as they are in the first few months of life if they are breast fed. It is proposed that this control over their own feeding may allow the infant who is following BLW to respond better to hunger and satiety cues than a baby who is spoon fed by someone else. It has also been pointed out that although the age at which it is recommended that parents start feeding their infant 'solids' has increased from 4 to 6 months of age, ${ }^{256}$ most countries have not changed their advice on how to introduce foods. ${ }^{3} 7$ The exception to this is the UK, 
where recent National Health Service (NHS) advice is that first foods can include soft vegetables and fruit offered as finger food or mashed. ${ }^{8}$

Despite increasing enthusiasm about BLW on the internet (8960 000 hits in May 2015) and in the social media, health governing bodies ${ }^{9}$ and some healthcare professionals $^{10}$ have expressed considerable concern that infants following a baby-led approach to infant feeding may be at an increased risk of choking and inadequate iron and energy intakes. Therefore, it is important to determine what infants following BLW are actually eating. The only study to date that has collected dietary information about BLW was a pilot study in just 11 families that focused on parental diet. Although some of the foods eaten by infants were reported, infant nutrient intakes were not analysed, and there was no comparison group of families following traditional spoon feeding (TSF). ${ }^{11}$

Therefore, the aims of the current study were: first, to determine whether there are differences in nutrient intakes and food intakes (particularly foods thought to pose a choking risk, foods high in iron, and foods containing added sugars or salt) between infants following BLW and those following TSF; and, second, to describe the 'family meals' offered to infants following BLW and TSF.

\section{METHODS}

Study design

This community-based cross-sectional study of infants aged 6-8 months combined data from three sources: two small cross-sectional studies (the 'Infant Feeding Study' and the 'How to Measure Infant Feeding' study), and the control group of a randomised controlled trial (the 'Baby-Led Introduction to SolidS' (BLISS) study). ${ }^{12}$ All participants completed a pretested demographic questionnaire and a feeding questionnaire, and a 3-day or 1-day weighed diet record (WDR) depending on the study. All adult participants provided written informed consent.

\section{Participants}

As many eligible participants as possible were recruited from the following three studies (with BLW and TSF infants being recruited from each study):

1. 'Infant Feeding Study' (December 2013 to June 2014), Dunedin and Auckland (New Zealand)advertisements in local newspapers and on social media sites, posters placed in a range of community areas, word of mouth in local parent support networks (29 mother-child pairs expressed interest, of whom 19 were eligible for this study);

2. 'How to Measure Infant Feeding' study (April 2013), Dunedin (New Zealand)-advertisements on social media sites, posters placed in a range of community areas, word of mouth in local parent support networks (11 participants were recruited, of whom 7 were eligible for this study);

3. BLISS study (September 2013 to June 2014), Dunedin (New Zealand) ${ }^{12}$-all women booking into the only birthing facility in Dunedin, the Queen Mary Maternity Unit, Dunedin Hospital, were invited to participate in the BLISS study ( $23 \%$ of those eligible volunteered). All those who were eligible for, and had consented to participate in, the BLISS study, had been randomised to the control group and had completed the WDR administered at 7 months of age at the time we were recruiting for this study were invited to contribute their data (40 participants were approached, of whom 25 consented to contribute their data and met the age-matching and sexmatching criteria (see below)).

Inclusion criteria for this study were: infant 6-8 months of age when the WDR was completed, mother able to communicate in English or Te Reo Māori (the language of the indigenous people of New Zealand) and mother 16 years of age or older. Exclusion criteria were: infant born before 37 weeks gestation; or presence of a congenital abnormality, physical condition or intellectual disability likely to affect the infant's feeding or growth. Infant participants were matched to within \pm 1 week of age and, wherever possible, for sex. BLISS study participants were used as a pool of prospective matches with the first match that was identified being recruited into this study.

\section{Definition of BLW and TSF}

Mothers were asked to state “...what approach to infant feeding you were using around the time you completed the food diary: 'Spoon-feeding' or 'Baby-Led Weaning' or 'Other'." Parents who reported following BLW were assigned to the full BLW group, whereas those who reported following a mixture of spoon feeding and BLW were assigned to the partial BLW group. Parents who were spoon feeding their child (without reporting BLW) were assigned to the TSF group.

\section{Questionnaires}

The same questions were asked of all participants. The demographic questionnaire collected information on: infant date of birth, sex, ethnicity (New Zealand Census questions ${ }^{13}$ ), birth weight and gestational age at birth; and maternal date of birth and parity. The feeding questionnaire collected data on: duration of exclusive breast feeding, ${ }^{2}$ age when complementary foods were introduced, extent of infant self-feeding versus parent feeding (and puréed vs finger foods) on the first complementary feeding occasion, ages when iron-fortified infant cereal and red meat were first introduced, and whether breast milk or infant formula was currently being consumed.

\section{Dietary assessment}

All three studies used the same WDR which was collected on three random non-consecutive days including two weekdays and one weekend day over a month (the 'Infant Feeding Study' and the BLISS study: $\mathrm{n}=44$ ) or on 1 day (the 'How to Measure Infant Feeding' study: $n=7$ ). 
The diet record had three key components: (1) a record of the foods eaten-time of day, type and brand of food or drink, preparation method, weight of food or drink, consistency of food or drink (puréed, mashed, diced, whole), who fed the child (parent, child, both), and total weight and estimated proportions of any leftover food or drink; (2) a description of any recipes usedraw amounts of ingredients, cooking method and proportion of the total recipe fed to the child and (3) an 'end of day questionnaire' which determined, for each meal and snack, whether the child ate with at least one adult, and whether the meal ingredients and preparation were the same as or different from the family meal. Resources were also provided to assist with estimating food portions when outside the home or in early childhood education.

Parents were given detailed oral and written instructions on how to complete the WDR, and were provided with a set of electronic scales (Salter Electronic Model 1017 , Kent, UK), accurate to within $\pm 1 \mathrm{~g}$.

All diet records were entered into the dietary analysis software program 'Kai-culator' V.1.11v (University of Otago, Dunedin, New Zealand). Kai-culator uses the New Zealand food composition database, FOODfiles; ${ }^{14}$ nutrient data for commonly consumed recipes collated in the 2008/2009 New Zealand Adult Nutrition Survey; ${ }^{15}$ and nutrient data for commercial infant foods calculated by the research team. ${ }^{16}$ Breast milk intake was assumed to be $750 \mathrm{~g} /$ day based on a quadratic curve fitted to the breast milk volumes reported by Dewey et $a l^{17}$ with the amount of infant formula consumed subtracted from this total if infants were mixed fed. After the diet records had been entered in Kai-culator, a registered dietitian blinded to the BLW or TSF status of the infant checked each diet record, and made corrections when required.

Diet record data were used to determine nutrient intake, the percentage of foods currently adult or self-fed, the percentage of foods currently fed as purées or finger foods, and whether any of the following foods were offered: foods thought to pose a choking risk (described below), iron-fortified infant cereal, red meat (defined as beef or lamb), foods with sugar added (ie, $>4 \mathrm{~g} / 100 \mathrm{~g}$ of added sugar or honey ${ }^{18}$ ), foods that were high in sodium (ie, $>350 \mathrm{mg}$ sodium $/ 100 \mathrm{~g}^{18}$ ), fruits, vegetables and commercial baby food. Foods thought to pose a choking risk were identified using lists from the literature ${ }^{19} 20$ and public health organisations, ${ }^{21} 22$ and advice from a paediatric speech-language therapist. Each WDR day was reviewed against this list to determine which foods thought to pose a choking risk were offered to infants.

\section{Statistical analysis}

The data were analysed using Stata V.13.1 (StataCorp, College Station, Texas, USA). A two-sided $\mathrm{p}<0.05$ was considered to indicate statistical significance.

Participant characteristics were examined for differences between the full BLW and TSF groups (table 1). Continuous variables (infant age, birth weight, gestational age and maternal age at birth) were compared using unpaired, two-tailed t tests. Categorical variables (infant

Table 1 Demographic and early feeding characteristics of participants according to method of complementary feeding ${ }^{*}$ (mean (SD) unless stated otherwise)

\begin{tabular}{|c|c|c|c|c|}
\hline & \multirow[b]{2}{*}{ TSF $(n=26)$} & \multicolumn{2}{|c|}{ BLW } & \multirow[b]{2}{*}{ p Valuef } \\
\hline & & Partial $(n=7)$ & Full $(n=18)$ & \\
\hline Infant age (months) & $7.3(0.7)$ & $7.3(0.4)$ & $7.4(0.8)$ & 0.690 \\
\hline Infant sex, $\mathrm{n}(\%)$ & & & & 0.540 \\
\hline Female & $12(46 \%)$ & $3(43 \%)$ & $10(56 \%)$ & \\
\hline Male & $14(54 \%)$ & $4(57 \%)$ & $8(44 \%)$ & \\
\hline Infant ethnicity, $\mathrm{n}(\%) \ddagger$ & & & & 0.383 \\
\hline NZ European & $20(77 \%)$ & $6(86 \%)$ & $11(65 \%)$ & \\
\hline Other & $6(23 \%)$ & $1(14 \%)$ & $6(35 \%)$ & \\
\hline Infant birth weight (grams) & $3528(419)$ & $3883(508)$ & $3288(400)$ & 0.071 \\
\hline Gestational age at birth (weeks) $\ddagger$ & $39.8(1.4)$ & $40.4(1.2)$ & $39.2(1.1)$ & 0.156 \\
\hline Maternal age at birth (years) & $33.9(4.4)$ & $35.1(2.8)$ & $33.1(3.7)$ & 0.542 \\
\hline Maternal parity, $\mathrm{n}(\%)$ & & & & 0.685 \\
\hline Primiparous & $4(15 \%)$ & $1(14 \%)$ & $2(11 \%)$ & \\
\hline Multiparous & $22(85 \%)$ & $6(86 \%)$ & $16(89 \%)$ & \\
\hline Mean duration of exclusive breast feeding (weeks) & $14.4(8.6)$ & $17.1(7.3)$ & $22.2(7.6)$ & 0.003 \\
\hline Number exclusively breast fed to 6 months, $\mathrm{n}(\%) \S$ & 0 & $1(14 \%)$ & $8(44 \%)$ & $<0.001$ \\
\hline Age when complementary foods were introduced (weeks) & $21.3(2.8)$ & $19.5(3.5)$ & $24.6(2.0)$ & $<0.001$ \\
\hline Number introduced to complementary foods before 6 months, $n(\%) \S$ & $25(96 \%)$ & $6(86 \%)$ & $9(50) \%$ & 0.001 \\
\hline
\end{tabular}


sex, ethnicity, maternal parity, feeding practices and when solids were introduced) were compared using $\chi^{2}$, and Fisher's exact tests where cell numbers were low $(<5)$.

Differences in BLW-associated behaviours between the full BLW and TSF groups when 'solids' were first introduced were determined using $\chi^{2}$ test for proportions (table 2). Since current feeding practices were skewed, medians and IQRs for each group were calculated and a Wilcoxon-Mann-Whitney test was used to determine if the groups differed.

The number of infants in each group who consumed a food type of interest (eg, foods thought to pose a choking risk) or a type of milk (eg, breast milk) on any day of the diet record was recorded and ORs with a $\chi^{2}$ test comparing the two groups were calculated (table 3 ).

Mean nutrient intake was calculated using the mean of all available days of the diet record for each participant (table 4). Since the data were mostly right-skewed, geometric means and 95\% CIs are presented. To determine the mean difference between groups in nutrient intake, all days of the diet record were used in a mixed-effects model with group as a fixed effect and participant identification number as a random effect. Log-transformed nutrient amounts were used as the outcome variable and regression coefficients backtransformed and presented as mean per cent difference between the groups along with $95 \%$ CIs and $p$ values.

The prevalence of inadequate zinc intakes was determined using the Estimated Average Requirement (EAR) cut-point method. ${ }^{23}$ Whether the group was likely to have adequate intakes of the other nutrients was determined by comparing the group mean intake to the adequate intake (AI). The AI cannot be used to calculate the prevalence of inadequate nutrient intake; however, when groups have a mean intake at or above the AI, it can generally be assumed that there is a low prevalence of inadequate nutrient intake for that population group. ${ }^{23}$

In table 5, for descriptive purposes, the number of infants in each group who shared meals with their family (eg, breakfast) was summed using the first day of the diet record. To compare consumption patterns between groups over all 3 days of the diet record, population-averaged generalised estimating equations for binary data were used with an exchangeable working correlation. Coefficients were back transformed to give ORs and 95\% CIs. This same technique was used to compare the number of infants who had the same or nearly the same meal ingredients and preparation as the family at mealtimes. An unstructured working correlation was used for these analyses.

\section{RESULTS}

\section{Participant characteristics}

Of the 80 families who were identified as potentially eligible to participate in the study, 13 were excluded because: their child was born before 34 weeks gestation $(n=2)$, their child was not aged between 6 and 8 months $(n=8)$ or the participant could not be contacted $(n=3)$; and 16 declined or were not required for age and sex matching. The final sample consisted of 26 infants reported to be following TSF and 25 reported to be following BLW either in part $(n=7)$ or fully $(n=18)$ at the time the diet record was collected. Mean (SD) maternal

Table 2 BLW-associated behaviours of infants when complementary foods were first introduced, and currently, according to method of complementary feeding

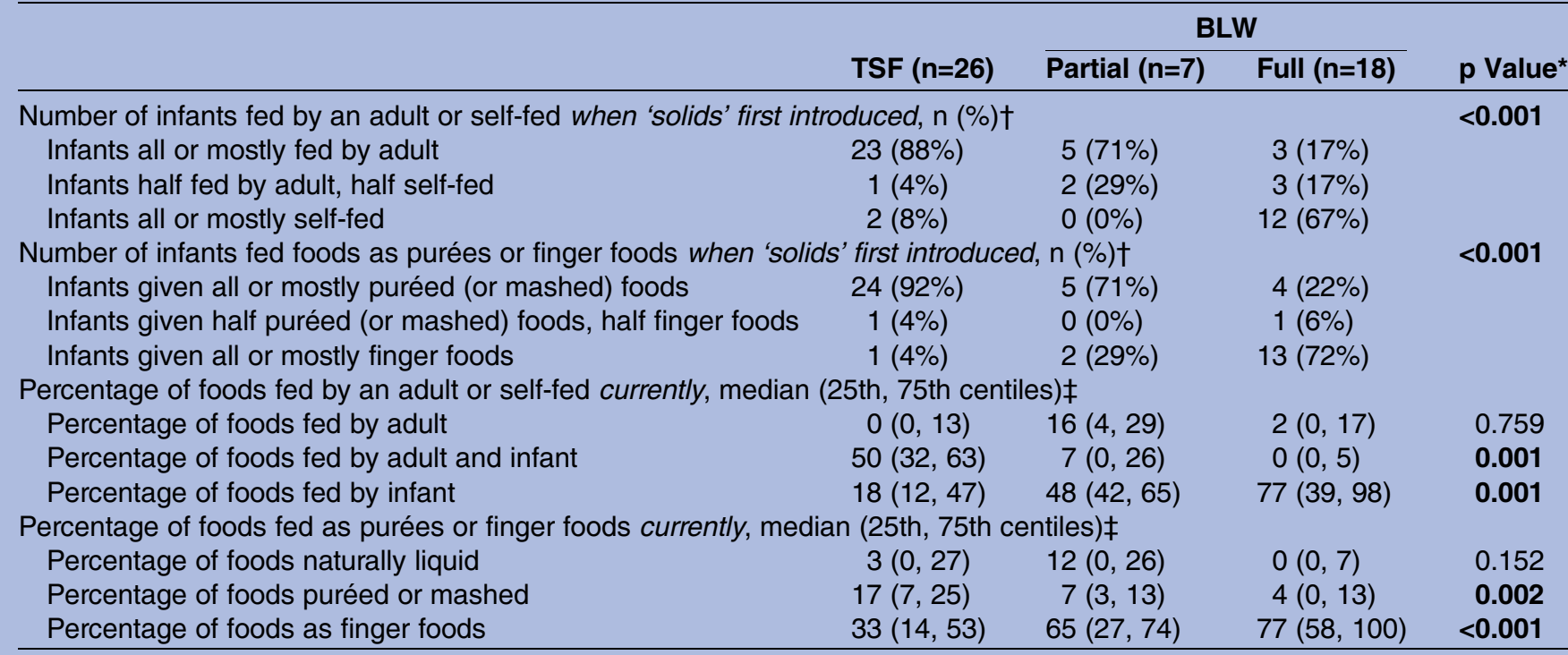

Bold typeface indicates a statistically significant difference $(p<0.05)$.

${ }^{*} p$ Values were calculated for differences between the full BLW and TSF groups using $\chi^{2}$ test for proportions, and Wilcoxon-Mann-Whitney test for median percentage of foods.

†Data from the feeding questionnaire.

$\ddagger$ Data from the weighed diet record collected when participants were between 6 and 8 months of age.

BLW, baby-led weaning; TSF, traditional spoon feeding. 
Table 3 Types of foods eaten by infants according to method of complementary feeding $(n(\%)$ unless stated otherwise)

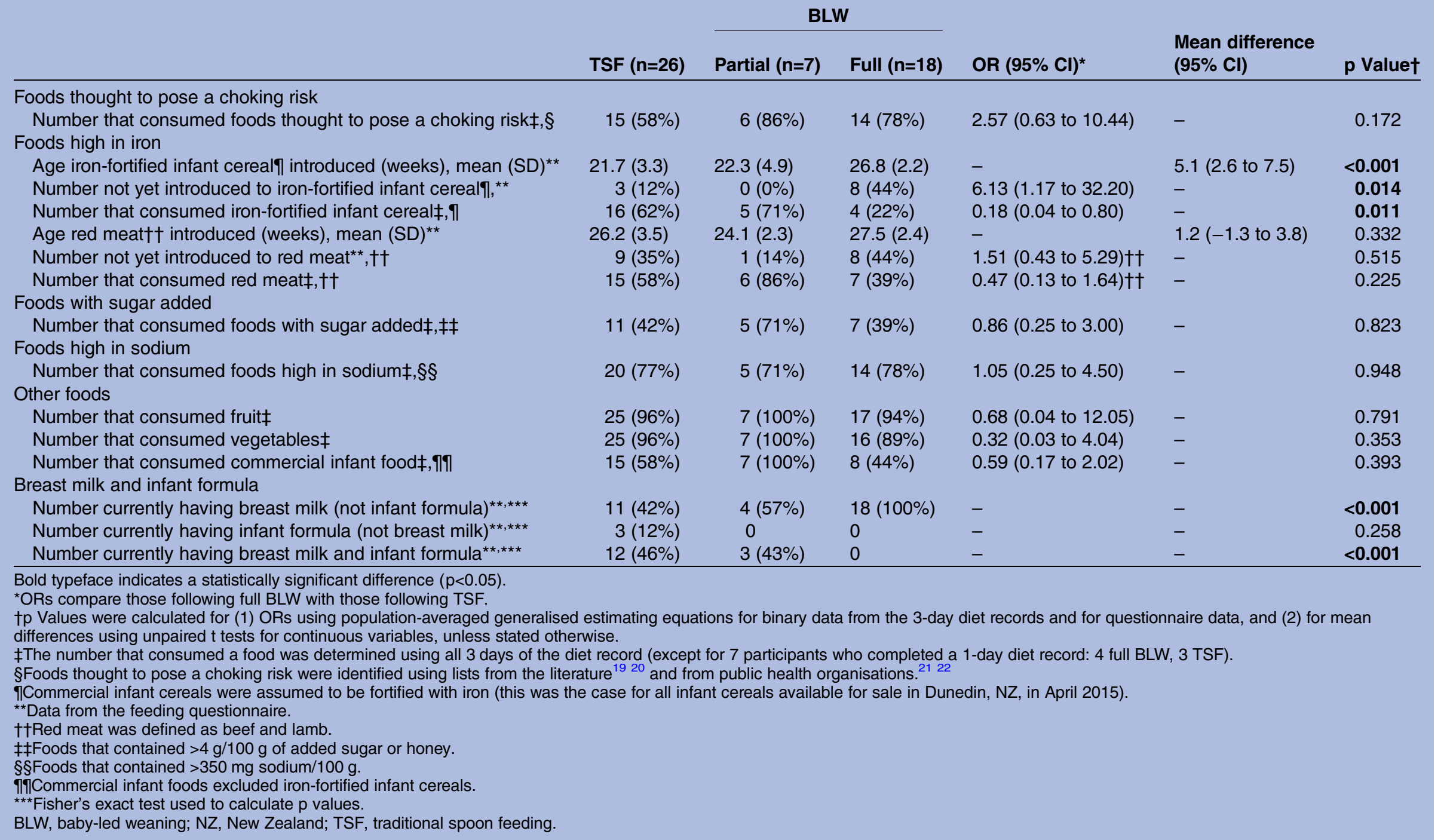


Table 4 Mean nutrient intake from weighed diet records of infants according to method of complementary feeding* (geometric mean (95\% CI))

\begin{tabular}{|c|c|c|c|c|c|c|}
\hline \multirow[b]{2}{*}{ Nutrients } & \multirow[b]{2}{*}{ Nutrient reference value† } & \multirow[b]{2}{*}{ TSF $(n=26) \ddagger$} & \multicolumn{2}{|c|}{ BLW } & \multirow{2}{*}{$\begin{array}{l}\text { Mean per cent difference } \\
\text { between groups } \$(95 \% \mathrm{Cl})\end{array}$} & \multirow[b]{2}{*}{ p Valueף } \\
\hline & & & Partial $(n=7)$ & Full (n=18)‡ & & \\
\hline Energy (kJ) & Boys: $2800^{\star \star} ;$ girls: $2500^{\star \star}$ & 2897 (2718 to 3088 ) & 3073 (2682 to 3521$)$ & 2800 (2518 to 3115$)$ & $-3.7(-13.5$ to 7.3$)$ & 0.500 \\
\hline Protein $(\mathrm{g})$ & $14 \mathrm{~g}$ & 17 (15 to 19$)$ & 18 (14 to 23 ) & 15 (12 to 17$)$ & $-14.4(-28.3$ to 2.3$)$ & 0.087 \\
\hline Protein (\% energy) & - & $10(9$ to 11$)$ & $10(9$ to 11$)$ & $9(8$ to 10$)$ & $-11.3(-20.6$ to -1.0$)$ & 0.033 \\
\hline Total fat $(\mathrm{g})$ & $30 \mathrm{~g}$ & 33 (31 to 35$)$ & 36 (33 to 38$)$ & 36 (33 to 39$)$ & 10.6 (0.4 to 21.9$)$ & 0.042 \\
\hline Total fat (\% energy) & - & 42 (39 to 44$)$ & 43 (39 to 47 ) & 48 (46 to 50$)$ & 15.1 (6.9 to 23.9$)$ & $<0.001$ \\
\hline Saturated fat $(\mathrm{g})$ & - & 14 (13 to 16$)$ & 16 (15 to 17$)$ & 17 (15 to 18$)$ & 15.6 (3.7 to 28.8$)$ & 0.009 \\
\hline Saturated fat (\% energy) & - & 18 (17 to 20$)$ & 19 (17 to 22$)$ & 22 (21 to 23$)$ & 20.0 (8.9 to 32.2$)$ & $<0.001$ \\
\hline Total carbohydrate $(\mathrm{g})$ & $95 \mathrm{~g}$ & 82 (75 to 90$)$ & 86 (70 to 105$)$ & 72 (64 to 82) & -12.1 (23.8 to 1.4$)$ & 0.076 \\
\hline Total carbohydrate (\% energy) & - & 48 (46 to 50$)$ & $47(44$ to 51$)$ & $44(42$ to 46$)$ & $-8.9(-14.0$ to -3.5$)$ & 0.001 \\
\hline Sugars $(g)$ & - & 46 (35 to 61$)$ & 54 (36 to 81$)$ & 62 (57 to 68$)$ & $42.3(-2.4$ to 107.3$)$ & 0.067 \\
\hline Dietary fibre $(\mathrm{g})$ & - & $3.6(2.2$ to 5.8$)$ & 3.7 (2.1 to 6.3$)$ & $2.0(1.2$ to 3.4$)$ & $-50.7(-73.3$ to -9.1$)$ & 0.023 \\
\hline Iron (mg) & $7 \mathrm{mg}$ & 3.6 (2.7 to 4.9$)$ & $3.3(1.3$ to 8.0$)$ & $1.6(1.2$ to 2.1$)$ & $-59.0(-72.5$ to -38.9$)$ & $<0.001$ \\
\hline Zinc (mg) & $2.5 \mathrm{mg} \dagger$ & 3.7 (3.3 to 4.1$)$ & $4.0(2.9$ to 5.4$)$ & $3.0(2.6$ to 3.3$)$ & $-20.5(-31.1$ to -7.4$)$ & 0.001 \\
\hline Vitamin C (mg) & $30 \mathrm{mg}$ & 66 (57 to 76$)$ & 67 (53 to 86$)$ & 46 (38 to 55$)$ & $-29.6(-40.0$ to -12.9$)$ & 0.001 \\
\hline Vitamin $B_{12}(\mu \mathrm{g})$ & $0.5 \mu \mathrm{g}$ & $0.5(0.3$ to 0.8$)$ & $0.6(0.3$ to 1.1$)$ & $0.2(0.1$ to 0.3$)$ & $-60.2(-76.2$ to -33.5$)$ & $<0.001$ \\
\hline Calcium (mg) & $270 \mathrm{mg}$ & 382 (352 to 436$)$ & 437 (311 to 616$)$ & 318 (290 to 349$)$ & $-19.1(-29.4$ to -7.3$)$ & 0.002 \\
\hline Sodium (mg) & $170 \mathrm{mg}$ & 235 (200 to 275$)$ & 235 (171 to 323$)$ & 232 (178 to 302 ) & $-1.5(-24.3$ to 28.2$)$ & 0.911 \\
\hline \multicolumn{7}{|c|}{ Bold typeface indicates a statistically significant difference $(p<0.05)$. } \\
\hline
\end{tabular}


Table 5 Relationship between the foods eaten by the infant and the meals eaten by their family (ie, family meals) according to method of complementary feeding $(n(\%))^{*}, \dagger$

\begin{tabular}{|c|c|c|c|c|c|}
\hline & \multirow[b]{2}{*}{ TSF $(n=26)$} & \multicolumn{2}{|c|}{ BLW } & \multirow[b]{2}{*}{ OR (95\% CI)‡ } & \multirow[b]{2}{*}{ p Values } \\
\hline & & Partial $(n=7)$ & Full $(n=18)$ & & \\
\hline \multicolumn{6}{|c|}{ Number of infants eating their meal with the family } \\
\hline Breakfast & $16 / 20(80 \%)$ & $6 / 6(100 \%)$ & $12 / 15(80 \%)$ & $1.99(0.48$ to 8.31$)$ & 0.344 \\
\hline Lunch & $13 / 21(50 \%)$ & $1 / 4(25 \%)$ & $12 / 14(86 \%)$ & 10.29 (2.67 to 39.65$)$ & 0.001 \\
\hline Evening meal & $12 / 23(52 \%)$ & $5 / 6(83 \%)$ & $12 / 15(80 \%)$ & $4.75(1.27$ to 17.75$)$ & 0.020 \\
\hline \multicolumn{6}{|c|}{ Number of infants with ingredients the same as the family meal } \\
\hline Breakfast & $5 / 19(19 \%)$ & $0 / 6(0 \%)$ & $5 / 14(36 \%)$ & $1.60(0.44$ to 5.78$)$ & 0.473 \\
\hline Lunch & $1 / 20(4 \%)$ & $0 / 6(0 \%)$ & $6 / 14(43 \%)$ & 10.56 (2.51 to 44.39$)$ & 0.001 \\
\hline Evening meal & $4 / 22(15 \%)$ & $0 / 6(0 \%)$ & $9 / 15(60 \%)$ & 9.00 (2.64 to 30.62$)$ & $<0.001$ \\
\hline \multicolumn{6}{|c|}{ Number of infants with meal preparation the same as the family meal } \\
\hline Breakfast & $2 / 19(8 \%)$ & $2 / 6(33 \%)$ & $6 / 13(46 \%)$ & 2.27 (0.59 to 8.70$)$ & 0.232 \\
\hline Lunch & $1 / 20(4 \%)$ & $0 / 3(0 \%)$ & $7 / 13(54 \%)$ & 10.31 (2.87 to 37.09$)$ & $<0.001$ \\
\hline Evening meal & $3 / 22(12 \%)$ & $1 / 6(17 \%)$ & $8 / 14(57 \%)$ & 8.18 (2.66 to 25.14$)$ & $<0.001$ \\
\hline \multicolumn{6}{|c|}{$\begin{array}{l}\text { Bold typeface indicates a statistically significant difference }(p<0.05) \text {. } \\
\text { *Summary numbers presented are from the first day of the diet record. } \\
\text { †Missing values: breakfast } n=10 \text {; lunch } n=12 \text {; evening meal } n=7 \text {. Most data were missing because: the infant was not offered the meal, only } \\
\text { infant milk was consumed at the meal or the infant was not with the parent for the meal. } \\
\text { fORs compare those following full BLW with those following TSF and are calculated using all } 3 \text { days of the diet record (except for } 7 \\
\text { participants who completed a 1-day diet record: } 4 \text { full BLW, } 3 \text { TSF). They may not therefore reflect exactly the same differences on the first } \\
\text { day of the diet record. } \\
\text { \$p Values were calculated for ORs using population-averaged generalised estimating equations for binary data. } \\
\text { IThe 'same as' was defined as the participant answering } 1=\text { exactly the same, or } 2=\text { almost the same, on a four-point scale (other values were } \\
3=\text { similar, } 4=\text { mostly different). } \\
\text { BLW, baby-led weaning; TSF, traditional spoon feeding. }\end{array}$} \\
\hline
\end{tabular}

age was 33.8 (3.9) years and most mothers $(86 \%)$ were multiparous. The infants were $6.0-8.8$ months of age and more than half were New Zealand European (74\%). There were no significant differences between the groups in any of the demographic variables collected (table 1).

\section{Feeding behaviours}

Women from the full BLW group exclusively breast fed their infants for $\sim 8$ weeks longer $(p=0.003)$, and introduced solid foods 3 weeks later $(\mathrm{p}<0.001)$, than women following TSF (table 1). In fact, $44 \%$ of the full BLW group exclusively breast fed their infant to 6 months, compared with none in the TSF group.

When complementary foods were first introduced, the TSF group was more likely $(\mathrm{p}<0.001)$ to have puréed or mashed food (92\% vs 22\%) and to be fed by an adult (88\% vs $17 \%$ ) than the full BLW group (table 2). Although the full BLW group was more likely to have finger foods as first foods ( $72 \%$ vs $4 \%$ ) which the infant self-fed $(67 \%$ vs $8 \%)$ than the TSF group, almost a third of participants in the full BLW group gave their infant puréed or mashed food $(28 \%)$ and a third fed their infant (rather than the infant feeding themself) when 'solids' were introduced. As would be expected, the partial BLW group was intermediate between the TSF and full BLW groups.

The diet record data suggest that when infants were 68 months of age, most infants in the full BLW group fed themselves more than half of their foods (a median of $77 \%$ of their foods were self-fed), whereas feeding was predominantly shared by the adult and infant in the TSF group (a median of $50 \%$ of food; $\mathrm{p}<0.001$; table 2 ).
Considerable differences were observed in the form in which foods were offered to the infants: while TSF babies consumed many finger foods (a median of $33 \%$ of their food intake was finger foods), this proportion was considerably lower than that observed in the full BLW group $(77 \% ; \mathrm{p}<0.001)$. At the time the study was conducted, parents reported that $50 \%$ of the full BLW infants were feeding themselves all their food, compared with $12 \%$ of those in the TSF group ( $p=0.015)$. Interestingly, $62 \%$ of parents following TSF reported that their infant was feeding themself some solids at least once a day (compared with $78 \%$ of those following full BLW; $\mathrm{p}=0.290$ ).

\section{Foods}

In total, two-thirds $(69 \%)$ of participants offered foods thought to pose a choking risk to their child, almost half (45\%) offered sweetened foods, and three-quarters (76\%) offered foods high in sodium on at least one occasion during the WDR; this did not differ between groups (table 3). However, although there was no statistically significant difference between the full BLW and TSF groups in the number of infants consuming foods thought to pose a choking risk $(78 \%$ vs $58 \%, \mathrm{p}=0.172)$, the CI for the OR was wide, so we cannot rule out higher odds of offering these foods in the full BLW group (OR, 95\% CI: 2.57, 0.63 to 10.44).

There were no differences in the number consuming fruit $(96 \%)$, vegetables $(94 \%)$ and, interestingly, the number using commercial baby foods (59\%). However, infants in the full BLW group were introduced to ironfortified infant cereal on average 5.1 weeks later than those following TSF, and had only one-fifth the odds of 
consuming iron-fortified infant cereal during the WDR. There were no differences in the age when red meat was introduced, the number not yet introduced to red meat (35\% overall), or the number who consumed it during the diet record.

Considerable differences were observed in breast milk and infant formula consumption between the full BLW and TSF groups, with significantly more participants in the full BLW group currently breast feeding (and not offering infant formula), than in the TSF group (100\% vs $42 \% ; \mathrm{p}<0.001)$. More participants in the TSF group than in the full BLW group were having both breast milk and infant formula ( $12 \%$ vs $0 \%, \mathrm{p}<0.001)$.

For those following full BLW, the most commonly consumed food types thought to pose a choking risk were: raw vegetables, raw apple and dried fruit (which were offered on 10, 3 and 3 days, respectively, of the 46 days recorded by participants in their WDRs), whereas for those following TSF, the most commonly consumed foods thought to pose a risk were: rusks, small pieces of meat (other than sausages and similar processed meats, or battered fish), crackers and corn kernels (which were offered on $10,5,4$ and 4 days, respectively, of the 72 days recorded by participants in their WDRs).

\section{Nutrients}

Table 4 shows significantly higher mean intakes of total fat, saturated fat and percentage energy from fat and saturated fat in the full BLW group. In contrast, mean intakes of iron, zinc, vitamin $\mathrm{B}_{12}$, vitamin $\mathrm{C}$, dietary fibre and calcium were lower in the full BLW group than in the TSF group. No differences in mean dietary intake of energy, sugar or sodium were detected between the two groups. The 95\% CI for energy suggests that, at most, the energy intake of infants following full BLW is likely to be $13 \%$ lower to $7 \%$ higher than that in those following TSF. For most nutrients, the intake of the partial BLW group appeared to be similar to that of the TSF group.

In the absence of anthropometric data (eg, body mass index), it is not possible to determine whether energy intake was adequate, although the mean intakes for both groups were higher than the Estimated Energy Requirement (EER). Overall, 13.7\% of participants had inadequate intakes of zinc ( 5 from the full BLW group, 2 from the TSF group and 0 from the partial BLW group). Study participants are likely to have sufficient intake of protein, fat, vitamin $\mathrm{C}$ and calcium because the mean intakes of these nutrients were higher than the AI. However, carbohydrate, iron and vitamin $B_{12}$ adequacy cannot be determined because the group mean intakes were below the AI. There is no nutrient reference value for dietary fibre at this age.

\section{Family meals}

The relationship between the foods consumed at the three main meals (breakfast, lunch and evening meal) by the infant and those consumed by the family is reported in table 5. BLW was associated with greater infant involvement in family meal times, with full BLW infants significantly more likely to sit with the family during lunch and evening mealtimes. However, breakfast eaters in all three groups were likely to share breakfast with the family $(83 \%$ of the infants consuming the meal). Compared with those following TSF, mothers following a full BLW approach were more likely to offer foods that were similar to or the same (both ingredients and preparation) as those eaten by the rest of the family at lunch and at the evening meal.

\section{DISCUSSION}

Our findings suggest that there may be several differences in foods, nutrients and eating behaviours between children following BLW and those following a more TSF approach. In this small study, BLW was associated with a number of health-related behaviours that would be expected to be beneficial: longer duration of exclusive breast feeding, later introduction of complementary foods and greater participation in family meals. However, the iron intakes of full BLW infants appeared to be even lower than those of infants following TSF, and intakes of zinc and vitamin $B_{12}$ may also be marginal. Although total energy intakes were similar, the sources of that energy differed with full BLW infants appearing to consume more saturated and total fat than TSF infants. It is clear that a high proportion of all three groups were consuming foods thought to pose a choking risk, and it is not possible, in this study, to exclude the possibility that infants following BLW infants may consume more of these foods.

The two major strengths of this study are (1) the recruitment of groups of infants following BLW and TSF who were closely matched for age and sex and other demographic variables and (2) the careful measurement of dietary intake, with information collected on all foods and drinks consumed for up to 3 days, taking into account leftovers. Furthermore, the collection of information on whether the infant fed themself, and the form the food was in, for each food item in the diet record, allowed us to determine the true extent of baby-led feeding in families who consider themselves to be following BLW or TSF.

The major limitation of our study was its small sample size that may not be representative of the wider population, and that decreased our ability to detect differences between the feeding styles. However, a number of statistically significant differences were detected between the two complementary feeding styles, and CIs have been reported which enable the reader to determine the magnitude of possible differences in the population as a whole for those variables that were not statistically significantly different. The maternal participants were older, ${ }^{25}$ and more likely to be multiparous, ${ }^{26}$ than typical New Zealand mothers. However, they were just as likely as New Zealand mothers to be of non-European ethnicity, ${ }^{27}$ and the participants in the TSF group had a similar very 
low rate of exclusive breast feeding to 6 months. ${ }^{27}$ Although there were some differences, therefore, between the study participants and the general public, the groups were closely matched for age, sex and the demographic variables measured, so differences in the diets of the infants studied are not likely to be due to differences in demography. Another limitation was the use of estimated breast milk volume. It was not feasible to use test weighing or stable isotopes to measure intake in each individual, and estimated volumes are commonly used in dietary studies in infants. ${ }^{28-30}$ It is also somewhat reassuring that the energy intakes in the full BLW and TSF groups were not statistically significantly different, and were also very similar (a difference of just $3.7 \%$.) If the breast milk value we used underestimated breast milk volume, then overall energy intake would be higher in BLW-this is unlikely as studies to date have suggested lower rather than higher BMI in infants following BLW. $^{31} 32$ If the breast milk value we used overestimated breast milk volume, then the differences in nutrient intakes would be even greater than what we have reported. However, it must be borne in mind that breast milk provided the majority of the energy intake in the BLW group ( $77 \%$ of energy), and that breast milk intake was not individually measured. Finally, families were assigned to the full BLW, partial BLW and TSF groups based on parental report of the complementary feeding approach used, rather than on an objective definition. Although this means that many in the full BLW group were not following the approach fully, the differences in feeding behaviours were substantial, and the use of the parents' categorisation enabled us to determine the dietary characteristics of infants whose parents would describe them as following BLW.

Three major concerns have been raised regarding the use of a baby-led approach to complementary feeding, namely whether the infant will consume sufficient energy and iron, and whether they will be at increased risk of choking. ${ }^{10}$ It has been proposed that infants may not have the motor skills or motivation to feed themselves enough food to meet their energy needs for growth if they are following BLW. ${ }^{10}$ This may be particularly relevant if low energy finger foods such as fruit and vegetables predominate in the diet. ${ }^{33}$ Since this study did not measure infant body mass index, growth faltering could not be identified, but the reported energy intakes were similar for the two feeding styles, were comparable to those reported for New Zealand infants ${ }^{34}$ and met the recommendations. ${ }^{24}$

In contrast, iron intakes appeared to be very different. Achieving adequate iron intake is problematic for infants worldwide because by 6 months of age substantial amounts of iron are needed from complementary foods. ${ }^{1} 3536$ Iron-fortified infant cereals are recommended as a suitable first food to help address this. ${ }^{2} 37$ It has been proposed that the BLW infant may be at particular risk of iron deficiency because the texture of infant cereal makes it difficult for infants to self-feed, and because foods that are easier to grasp tend to be naturally low in iron (eg, fruits and vegetables). ${ }^{10}$ Our results would suggest that these concerns may have some value given that, on average, infants following full BLW appeared to have less than half the daily dietary iron intake of infants following TSF. The full BLW group introduced fortified infant cereal 5 weeks later and were considerably less likely to consume fortified infant cereal during the WDR. Although the adequacy of intakes below the AI cannot be determined, ${ }^{23}$ the iron intakes of all three groups were considerably lower than the AI of $7 \mathrm{mg}^{24}$ (3.6, 3.3 and $1.6 \mathrm{mg}$ for TSF, partial BLW and full BLW, respectively). Half of the infants (51\%) consumed no fortified infant cereal over the 3 days of WDR, $45 \%$ consumed no red meat and $22 \%$ consumed neither fortified cereal nor red meat. It is important that these results are confirmed in other studies, particularly studies determining iron status as well as intake, so that it can be determined whether infants following BLW have poorer iron status. In the meantime, healthcare professionals should emphasise the importance of including iron-rich food sources in infants' diets in the complementary feeding period because of the wellaccepted challenges of achieving adequate iron intake at this age, whether BLW or TSF is being followed. It is important to note that it is possible that the choice of infant milk may have contributed to these differences in total iron intake-none of the infants in the full BLW group were having infant formula, whereas more than half were consuming formula in the TSF group $(n=15$; $58 \%$ ), and infant formulas have a higher iron concentration than breast milk.

It has been suggested that infants following BLW may be at increased risk of choking because they are feeding themselves whole foods during the early stages of complementary feeding, while they are still learning to chew and swallow. ${ }^{10} 38$ Although our sample size was too small to investigate actual choking incidents, we observed that a worryingly high number of parents in all three groups were offering foods thought to pose a choking risk. These results suggest that further education for parents on how to minimise the risk of choking may be needed-no matter what approach to complementary feeding is being used. This advice would need to refer not just to types of food that are commonly considered to pose a choking risk (eg, one parent offered their infant whole nuts), but also to foods that do not pose a risk to older children and adults so may form part of the family meal (eg, corn); and to ways in which foods thought to pose a choking risk can be modified to make them safer (eg, chicken is safer if it is offered in pieces that can be chewed on but not put in the mouth whole, or if it is chopped finely). It is not clear from our study sample whether the population of infants following BLW is more likely to be exposed to foods thought to pose a choking risk. Although there was no statistically significant difference between the groups, the CI for the OR was wide, so we cannot rule out higher odds of 
exposure in the full BLW group. It is therefore extremely important that exposure to foods thought to pose a choking risk, and choking prevalence, are investigated in future larger studies of BLW.

Concern has been expressed that infant self-feeding of family meals is only of benefit if the family meals themselves are nutritionally adequate. ${ }^{10}$ While previous studies have reported that infants following BLW are more likely to eat with their family, ${ }^{33} 38{ }^{40}$ we extend these findings to show that the foods eaten also tend to more closely resemble those eaten by the family. Thus, it appears that BLW infants do follow a more 'adult' food pattern. This may be an issue given that exposure to a variety of 'unhealthy' family foods might lead to negative impacts on eating behaviours later in life. ${ }^{41}$ Certainly, in our study, total and saturated fat intakes were significantly higher for those following full BLW. It is recommended that total fat intakes should be $30-45 \%$ of energy, ${ }^{1}$ and the mean intake of those in the full BLW group appeared to be slightly above this $(48 \%$ of energy). It is not clear, however, whether the saturated fat intakes are of concern. Both the TSF (18\% energy) and BLW (22\% energy) saturated fat intakes are lower than mature breast milk, which has $25 \%$ energy from saturated fat. ${ }^{14}$ It is also possible that family meals do not provide sufficient zinc and vitamin $\mathrm{B}_{12}$ to meet the relatively high requirements of infants, particularly given the small portion sizes consumed by infants. There is considerable controversy about the EAR for zinc for infants, ${ }^{42}$ but $28 \%$ of the infants in the full BLW group in this study appeared to have a zinc intake less than the EAR of $2.5 \mathrm{mg}$ for Australian and New Zealand infants. ${ }^{24}$ It is not possible to state that the mean vitamin B12 intake in the BLW group of $0.2 \mu \mathrm{g} /$ day is inadequate, even though it is below the $\mathrm{AI} ;{ }^{23}$ however, intakes would need to more than triple by the age of 12 months to meet the EAR for that age $\left(0.7 \mu \mathrm{g} /\right.$ day $\left.^{24}\right)$. There were a number of other differences in nutrient intakes between the full BLW infants and those using TSF, but these are of less concern. Although the percentage energy from protein and carbohydrate was lower in the full BLW group, the gram amounts eaten were not significantly different, and although vitamin $\mathrm{C}$ and calcium intakes were lower, they were still well above the AI. Dietary fibre intake was also significantly lower in the full BLW group, but no recommended intake has been set for this age group because breast milk does not contain dietary fibre, and there are 'no functional criteria for dietary fibre in infants'. ${ }^{43}$ It is important to note when interpreting these dietary data that our study was small, crosssectional and not from a random sample of families, and that we cannot determine whether these differences would still be apparent at older ages, given that by 12 months of age, all children should be eating predominantly family foods. ${ }^{2}$ If confirmed, these results suggest that the current complementary feeding guidelines for infants, which were after all developed to meet the needs of traditionally spoon-fed infants, may need to be modified to account for parents who choose to follow BLW. Modifications would most likely include an emphasis on the importance of the family adopting healthy eating behaviours with a variety of nutrientdense foods that both the family and the infant can enjoy, and the inclusion of foods that are rich sources of iron, zinc and vitamin $\mathrm{B}_{12}$.

In conclusion, this study suggests that BLW may be associated with a number of health-related behaviours that would be expected to be beneficial: exclusive breast feeding to 6 months, waiting until 6 months to introduce solids and greater involvement in family meals; however, further research is required to determine whether BLW infants may be at higher risk of iron, zinc and vitamin $\mathrm{B}_{12}$ deficiencies. It would also be useful to determine the relative importance of the delayed introduction of solids and the high breastfeeding rates seen in infants following BLW, compared with BLW itself, in determining nutrient intakes in 6-8-month olds. The extent to which differences in total and saturated fat intake remain at 12 months also needs to be determined. Although BLW children did not appear to be offered more foods thought to pose a choking risk, it is of concern that two-thirds of the infants were offered at least one food thought to pose a choking risk during the diet record, and we cannot rule out the possibility that those following BLW may be more likely to offer such foods. Given the widespread interest and debate regarding the suitability of BLW as an alternative infant feeding method, further research in a larger, ideally representative, sample of children, preferably with measurements of growth, biochemical nutrient status and rate of choking, is required to confirm these findings. In the meantime, our findings suggest that families of infants following BLW should be encouraged to include a variety of nutrient-dense foods in family meals, and to offer their infants foods rich in iron, zinc and vitamin $\mathrm{B}_{12}$; and that all parents, no matter what approach they take to complementary feeding, should be given advice on how to minimise their infant's exposure to foods thought to pose a choking risk-ideally by changing methods of food preparation rather than by excluding foods, many of which make an important contribution to the diet if offered in safe shapes and textures.

Contributors A-LMH and RWT conceived the idea for the study and designed the research. BJM, CJS, AL, LWE and EAF collected the data and analysed the diet records. LJF designed the approach used to determine the consumption of foods thought to pose a choking risk. JJH completed all statistical analyses. BJM wrote the first draft of the manuscript. All authors contributed to the interpretation of the results and revision of the manuscript, and approved the final manuscript. A-LMH is the guarantor.

Funding This work was supported by the University of Otago departmental funds, a Department of Medicine Summer Research Scholarship (BJM), and the Department of Human Nutrition MDiet fund (CJS). RWT is supported by the Karitane Fellowship in Early Childhood Obesity.

Competing interests All authors have completed the ICMJE uniform disclosure form at http://www.icmje.org/coi_disclosure.pdf and declare: grants from Lottery Health Research, Meat \& Livestock Australia, The New Zealand 
Women's Institute, Karitane Products Society, and Perpetual Trustees-that is, agencies that funded the BLISS study - during the conduct of the work; outside the submitted work, RWT is a 'Perspectives: news and views' advisory board member, and part of her salary is from a Karitane Products Society (KPS) Fellowship that is paid via the University of Otago.

Ethics approval University of Otago Human Ethics Committee (Reference 13/270), and from the Lower South Regional Ethics Committee (Reference LRS/11/09/037).

Provenance and peer review Not commissioned; externally peer reviewed.

Data sharing statement No additional data are available.

Open Access This is an Open Access article distributed in accordance with the Creative Commons Attribution Non Commercial (CC BY-NC 4.0) license, which permits others to distribute, remix, adapt, build upon this work noncommercially, and license their derivative works on different terms, provided the original work is properly cited and the use is non-commercial. See: http:// creativecommons.org/licenses/by-nc/4.0/

\section{REFERENCES}

1. World Health Organization. Infant and young feeding: model chapter for medical students and allied health professionals. Geneva: World Health Organization, 2009.

2. New Zealand Ministry of Health. Food and Nutrition Guidelines for healthy infants and toddlers (aged 0-2): a background paper. 4th edn. Wellington: New Zealand Ministry of Health, 2012.

3. Cameron S, Heath AL, Taylor R. How feasible is baby-led weaning as an approach to infant feeding? A review of the evidence. Nutrients 2012;4:1575-609.

4. Rapley G, Murkett T. Baby-led weaning: helping your baby love good food. London: Vermillion, 2008.

5. World Health Organization. Guiding principles of complementary feeding of the breastfed child. Geneva: World Health Organization 2004.

6. World Health Organization. Guiding principles for feeding non-breastfed children 6-24 months of age. Geneva: World Health Organization, 2005

7. Rapley G. Baby-led weaning: transitioning to solid foods at the baby's own pace. Community Pract 2011;84:20-3.

8. National Health Service. Introducing solid foods: giving your baby a better start in life. Solid foods leaflet v0_8. Department of Health, 2014.

9. New Zealand Ministry of Health. Baby-led weaning-Ministry position statement. 2012 (accessed 25 Sep 2015). http://www.health. govt.nz/our-work/preventative-health-wellness/nutrition/baby-ledweaning-ministry-position-statement

10. Cameron S, Heath A-L, Taylor R. Healthcare professionals' and mothers' knowledge of, attitudes to and experiences with, baby-led weaning: a content analysis study. BMJ Open 2012;2:e001542.

11. Rowan $\mathrm{H}$, Harris $\mathrm{C}$. Baby-led weaning and the family diet. A pilot study. Appetite 2012;58:1046-9.

12. Daniels L, Heath AL, Williams S, et al. Baby-Led Introduction to SolidS (BLISS) study: a randomised controlled trial of a baby-led approach to complementary feeding. BMC Pediatr 2015;15:17.

13. Statistics New Zealand. 2013 Census. 2014 (accessed 25 Sep 2015). http://www.stats.govt.nz/Census/2013-census.aspx

14. New Zealand Ministry of Health. New Zealand food composition database (FOODfiles) 2013. (accessed 25 Sep 2015). http://www. foodcomposition.co.nz/foodfiles

15. New Zealand Ministry of Health, University of Otago. Methodology report for the 2008/09 New Zealand Adult Nutrition Survey. Wellington: New Zealand Ministry of Health, 2011.

16. Clouston A. The role of commercial processed baby foods in the diets of New Zealand toddlers [MDiet thesis]. University of Otago, 2014.

17. Dewey $\mathrm{K}$, Heinig $\mathrm{M}$, Nommsen $\mathrm{L}$, et al. Adequacy of energy intake among breast-fed infants in the DARLING study: relationships to growth velocity, morbidity, and activity levels. $J$ Pediatr 1991:119:538-47.

18. Food Standards Australia New Zealand. Food Standards CodeStandard 2.9.2-Foods for Infants. Canberra: Food Standards Australia New Zealand, 2014.
19. Cameron S, Taylor R, Heath AL. Development and pilot testing of Baby-Led Introduction to SolidS-a version of baby-led weaning modified to address concerns about iron deficiency, growth faltering and choking. BMC Pediatr 2015;15:99.

20. Nichols B, Visotcky A, Aberger M, et al. Pediatric exposure to choking hazards is associated with parental knowledge of choking hazards. Int J Pediatr Otorhinolaryngol 2012;76:169-73.

21. New Zealand Ministry of Health. Preventing choking in young children. 2014 (accessed 25 Sep 2015). http://www.health.govt.nz/ your-health/healthy-living/food-and-physical-activity/nutrition/foodrelated-choking-young-children/preventing-choking-young-children

22. American Academy of Pediatrics. Choking prevention. 2015 (accessed 22 Sep 2015) https://www.healthychildren.org/English/ health-issues/injuries-emergencies/Pages/Choking-Prevention.aspx

23. Institute of Medicine. Dietary reference intakes: applications in dietary assessment. Washington DC: National Academy Press, 2000.

24. National Health and Medical Research Council of Australia, New Zealand Ministry of Health. Nutrient reference values for Australia and New Zealand. Canberra, Australia: National Health and Medical Research Council of Australia, 2006.

25. Statistics New Zealand. Births and deaths: year ended December 2013. Wellington: Statistics New Zealand, 2014.

26. Morton S, Atatoa Carr P, Grant C, et al. Growing up in New Zealand: a longitudinal study of New Zealand children and their families. Report 2: now we are born. Auckland, New Zealand: Growing Up in New Zealand, 2012.

27. Statistics New Zealand. Census QuickStats about a place: Dunedin City. 2014. Retrieved 21 January 2016 http://wwwstatsgovtnz/ Census/2013-census/profile-and-summary-reports/quickstats-abouta-placeaspx? request value $=15022 \&$ tabname $=$ Culturaldiversity

28. Devaney B, Kalb L, Briefel R, et al. Feeding Infants and Toddlers Study: overview of the study design. J Am Diet Assoc 2004;104: s8-13.

29. Sharma S, Kolahdooz F, Butler L, et al. Assessing dietary intake among infants and toddlers $0-24$ months of age in Baltimore, Maryland, USA. Nutr J 2013;12:52.

30. Skinner J, Carruth B, Houck K, et al. Longitudinal study of nutrient and food intakes of infants aged 2 to 24 months. J Am Diet Assoc 1997;97:496-504

31. Brown A, Lee M. Early influences on child satiety-responsiveness: the role of weaning style. Pediatr Obes 2015;10:57-66.

32. Townsend E, Pitchford N. Baby knows best? The impact of weaning style in food preferences and body mass index in early childhood in a case-controlled sample. BMJ Open 2012;2:e000298.

33. Brown $A$, Lee M. A descriptive study investigating the use and nature of baby-led weaning in a UK sample of mothers. Matern Child Nutr 2011;7:34-47.

34. Soh P, Ferguson E, McKenzie J, et al. Dietary intakes of 6-24month-old urban South Island New Zealand children in relation to biochemical iron status. Public Health Nutr 2002;5:339-46.

35. Grant $\mathrm{C}$, Wall $\mathrm{C}$, Brunt $\mathrm{D}$, et al. Population prevalence and risk factors for iron deficiency in Auckland, New Zealand. J Paediatr Child Health 2007;43:532-8.

36. Soh P, Ferguson E, McKenzie J, et al. Iron deficiency and risk factors for lower iron stores in 6-24-month-old New Zealanders. Eur J Clin Nutr 2004;58:71-9.

37. Domellöf M. Iron requirements in infancy. Ann Nutr Metab 2011;59:59-63.

38. Brown A, Lee M. An exploration of experiences of mothers following a baby-led weaning style: developmental readiness for complementary foods. Matern Child Nutr 2013;9:233-43.

39. Byard R, Gallard V, Johnson A, et al. Safe feeding practices for infants and young children. J Paediatr Child Health 1996;32:327-9.

40. Cameron S, Taylor R, Heath AL. Parent-led or baby-led? Associations between complementary feeding practices and health-related behaviours in a survey of New Zealand families. BMJ Open 2013;3:e003946.

41. Birch L. Development of food preferences. Annu Rev Nutr 1999;19:41-62.

42. Gibson R, Heath A-L. Population groups at risk of zinc deficiency in Australia and New Zealand. Nutr Diet 2011;68:97-108.

43. National Health and Medical Research Council of Australia, New Zealand Ministry of Health. Nutrient reference values for Australia and New Zealand including recommended dietary intakes. Canberra: National Health and Medical Research Council of Australia, 2006. 